\title{
Regard sur le modèle agricole sénégalais : pratiques foncières et particularités territoriales des moyennes et grandes exploitations agricoles
}

\author{
Jérémy Bourgoin ${ }^{1,2, *}$, Djibril Diop ${ }^{3}$, Djiby Dia ${ }^{3}$, Moussa Sall ${ }^{3}$, Romaric Zagré $^{3}$, Quentin Grislain ${ }^{1,2}$ \\ et Ward Anseeuw ${ }^{4,5,6}$ \\ 1 CIRAD, UMR TETIS, 34398 Montpellier, France \\ 2 TETIS, Univ. Montpellier, AgroParisTech, CIRAD, CNRS, IRSTEA, Montpellier, France \\ ${ }^{3}$ Institut sénégalais de recherches agricoles, Bureau d'analyses macro-économiques, Dakar, Sénégal \\ ${ }^{4}$ CIRAD, UMR ART-DEV, 34398 Montpellier, France \\ 5 ART-DEV, Univ. Montpellier, CIRAD, 34000 Montpellier, France \\ ${ }^{6}$ International Land Coalition, Rome, Italie
}

\begin{abstract}
Résumé - L'enjeu alimentaire lié à l'augmentation démographique est au cœur des agendas internationaux de développement durable et nourrit les débats sur les transitions à entreprendre ou soutenir. Souvent défini en opposition à un modèle agro-industriel intensif et capitaliste, le modèle des agricultures familiales, ensemble de types d'exploitations et d'exploitants aux profils variés, reste difficile à considérer dans sa diversité du fait de sa constante évolution. Certains phénomènes restent peu étudiés, notamment la dynamique d'accumulation foncière d'exploitations agricoles identifiée dans certains pays d'Afrique subsaharienne. Notre contribution, basée sur des enquêtes menées au Sénégal, vise à caractériser les profils et les modalités d'accès au foncier de 1399 exploitations agricoles, concentrant le plus de terres. À l'échelle de différentes zones agro-écologiques, nous mettons en évidence que les chefs d'exploitations agricoles possédant plus de 3 hectares sont majoritairement issus de la localité d'origine, cultivent des terres familiales et investissent dans l'agriculture des revenus issus de cette même activité. Globalement, ces exploitations agricoles ne semblent pas inféodées à une dynamique de gentrification, caractérisée par les investissements d'élites urbaines et rurales, souvent non issues du monde agricole, et faisant l'acquisition de terres grâce à des revenus non agricoles. Concernant les dynamiques foncières, les enquêtes montrent que la majorité des terres reste sous occupation coutumière bien que les territoires ruraux soient en constante évolution. Dans un contexte où les pouvoirs publics insistent sur les besoins d'intensification agricole, à travers la promotion de l'entreprenariat privé et du secteur agro-industriel, il est crucial d'avoir une bonne compréhension de la diversité des formes d'exploitations agricoles. Cela est d'autant plus nécessaire qu'une réforme foncière est en cours et qu'elle contribuera à redéfinir les contours du modèle agricole, entre volontés productivistes et exigences de durabilité et d'équité.
\end{abstract}

Mots clés : Sénégal / agriculture / accès à la terre / droits fonciers / politique foncière

Abstract - A look at the Senegalese agricultural model: land practices and territorial specificities of medium and large-scale farms. The food issue related to population growth is at the heart of international sustainable development agendas and feeds the debates on the transitions to be undertaken or supported. Often defined in opposition to an intensive and capitalist agro-industrial pathway, the family farming model, which encompasses a set of farm types and farmers with varied profiles, remains difficult to consider in all its diversity due to its constant evolution. Some phenomena remain little studied, in particular the dynamics of land accumulation on farms identified in some sub-Saharan African countries. Our contribution, based on surveys conducted in Senegal, aims to characterize the profiles and modalities of access to land of 1399 farms, with the highest land concentration. At the scale of different agro-ecological zones, we show that the heads of farms with more than 3 hectares are mostly originating from the locality, cultivate family

\footnotetext{
* Auteur de correspondance : jeremy.bourgoin@cirad.fr
} 
land and invest in agriculture income from this same activity. On the whole, these farms do not seem to be subject to a gentrification dynamic, characterized by investments by urban and rural elites, often not coming from the agricultural world, and who acquire land with non-agricultural income. With regard to land dynamics, surveys show that the majority of land remains under customary occupation although rural territories are in constant evolution. In a context where public authorities are emphasizing the need for agricultural intensification, through the promotion of private entrepreneurship and the agro-industrial sector, it is crucial to have a good understanding of the diversity of farm forms. This is all the more necessary as a land reform is under way and will help redefine the contours of the agricultural model, between productivity and the demands of sustainability and equity.

Keywords: Senegal / agriculture / land access / land rights / land policies

\section{Introduction}

Au Sénégal, comme dans la majeure partie des pays d'Afrique de l'Ouest, les stratégies nationales de développement considèrent l'appui à l'agriculture familiale comme le principal moyen d'atteindre des objectifs de croissance agricole à même de répondre aux enjeux actuels et futurs de sécurité et souveraineté alimentaire (NEPAD, 2003). Cependant, les moyens mis à disposition des exploitations agricoles sont rarement en adéquation avec les discours portés (Sourisseau, 2014). En compétition avec d'autres formes de modèles agricoles et d'autres secteurs d'activité dans un contexte de libéralisation des marchés (Fig. 1), les exploitations agricoles familiales souffrent aussi d'un manque de reconnaissance dans leur diversité, sous-représentée dans les statistiques nationales (Barral et al., 2014). Souvent défini en opposition à un modèle agro-industriel intensif et capitaliste, le modèle des agricultures familiales est pourtant un ensemble diversifié d'exploitations aux caractéristiques variées (Sourisseau et al., 2012). La mise en évidence de nouvelles dynamiques, comme l'accumulation foncière de la part d'investisseurs de taille moyenne, identifiée par Jayne et al. (2014) dans certains pays africains, nécessite d'approfondir les recherches sur les exploitations agricoles. Au Sénégal, cette dynamique n'a pas fait l'objet d'études spécifiques à ce jour et les recensements disponibles pour le secteur agricole datent de 1998. Pourtant, des éléments de diagnostic quantifiant son ampleur et ses caractéristiques seraient essentiels au processus de réforme foncière relancé depuis 2012. Piloté par la Commission nationale de réforme foncière (CNRF), ce processus a abouti en 2016 à l'élaboration d'une proposition de politique foncière. Au-delà de grands principes et orientations visant à sécuriser un accès équitable à la terre, le document de politique foncière ne permet pas de nuancer les considérations d'accès à la terre au regard de la diversité d'acteurs qui composent le monde agricole (CNRF, 2016). En complément des travaux d'identification des dynamiques foncières des exploitations agro-industrielles à capitaux étrangers (Bourgoin et al., 2019) et d'analyses structurelles des exploitations familiales (FONGS, 2013), notre contribution vise à caractériser les profils et les modalités d'accès au foncier d'exploitations concentrant le plus de terres. Nos travaux se basent sur des enquêtes menées auprès de 1399 exploitations agricoles sénégalaises de plus de 3 hectares (excluant les agro-industries ou exploitations à capitaux étrangers), sélectionnées dans une base de sondage de plus de 8900 exploitations constituée au préalable et identifiant les caractéristiques d'exploitations réparties dans différentes zones agro-écologiques du pays. La sélection des exploitations à étudier s'est faite sur la base des superficies possédées pour permettre l'analyse du lien entre concentration foncière et modalités d'accès à la terre. Les résultats identifiant les profils et les stratégies d'accès et d'usage des terres de ces exploitations nous permettent dans une dernière partie de discussion de mettre en perspective les pratiques foncières observées et leurs particularités issues de dynamiques territoriales spécifiques.

\section{Méthodes}

\subsection{Contexte agricole et foncier au Sénégal}

Au Sénégal, l'agriculture représente le principal secteur d'emploi et mobilise près de $70 \%$ de la population active. Son poids dans le secteur primaire est passé de $41 \%$ en 2007 à $64 \%$ en 2017 (RCSA, 2017). La contribution de ce secteur au produit intérieur brut est en légère augmentation sur cette même période, passant de $13 \%$ à $15 \%$. Les derniers recensements de population montrent que $70 \%$ des exploitations agricoles sont de petites exploitations familiales dont les superficies sont inférieures à 5 hectares (ANSD, 2014). La poursuite de la croissance du secteur agricole est mise en œuvre par les pouvoirs publics au travers de programmes sectoriels, comme le récent Programme d'accélération de la cadence de l'agriculture sénégalaise de 2013. La gestion du foncier au Sénégal, notamment en zone rurale, est régie par la loi $n^{0}$ 64-46 du 17 juin 1964 relative au domaine national (DN), qui fait l'originalité du système foncier sénégalais. Les terres n'appartiennent ni à l'État, ni aux collectivités territoriales, ni aux usagers, mais à la « Nation ». Elles sont inaliénables, intransmissibles, incessibles et elles ne peuvent être transférées que dans le domaine privé de l'État par voie d'immatriculation. Cette opération donne ensuite accès à des droits réels (e.g. titre foncier ou bail). Les collectivités locales ont la responsabilité de l'affectation/désaffectation des terres du domaine national pour les activités rurales. Jamais réformé, ce système est aujourd'hui remis en cause en raison de sa faible capacité à sécuriser les investissements étrangers et de l'impossibilité du développement d'un marché foncier officiel (CNRF, 2016).

\section{2 Échantillonnage et collecte de données}

L'étude de terrain réalisée au Sénégal se base sur les cadres conceptuels développés par Anseeuw et al. (2016) 


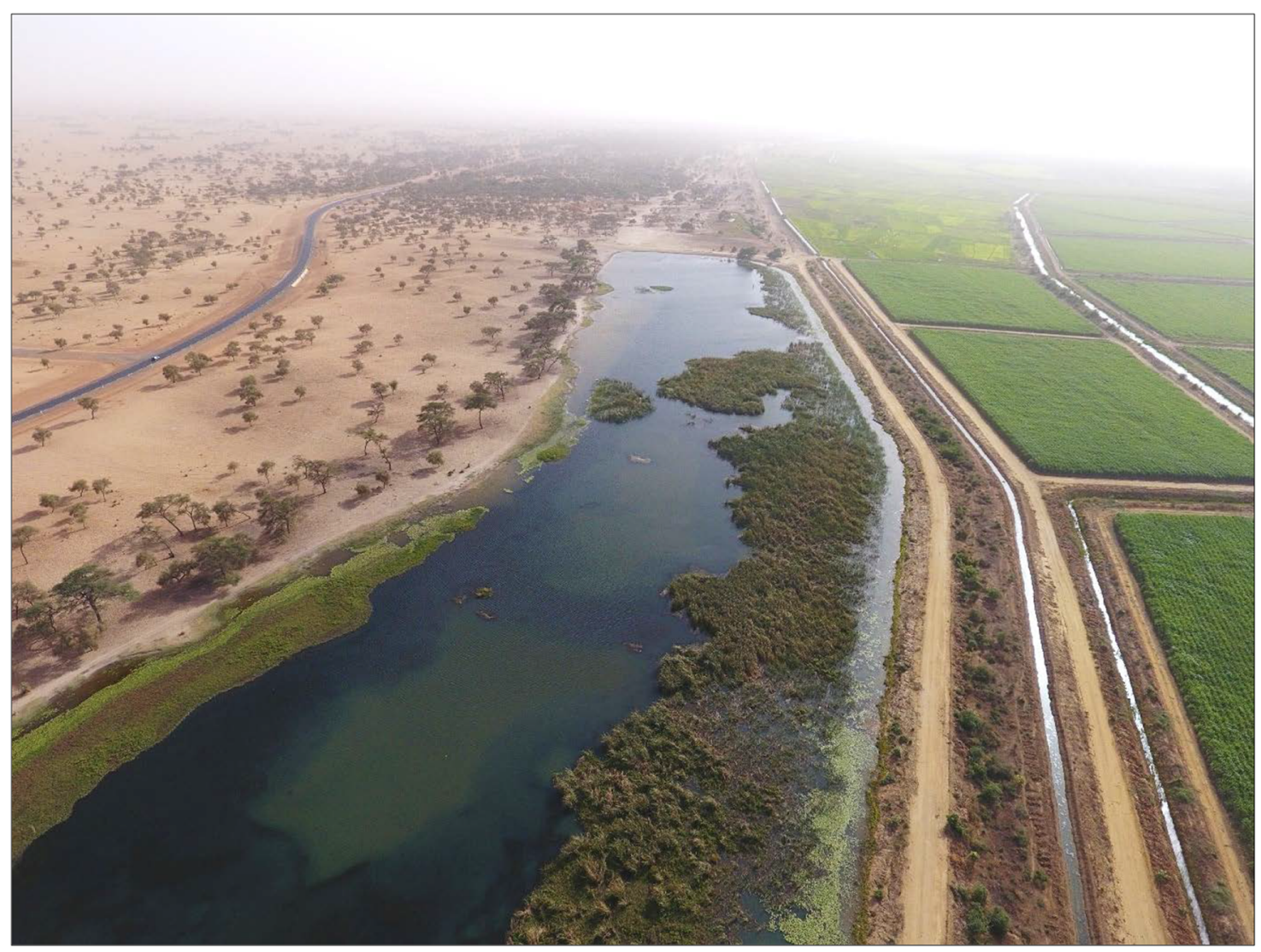

Fig. 1. Mise en évidence de différentes formes de modèles agricoles dans le Delta du fleuve Sénégal.

Fig. 1. Highlighting different forms of agricultural models in the Senegal River Delta.

et Jayne et al. (2016), associant l'analyse des modèles d'accumulation de terres et des trajectoires agricoles pour l'étude socio-économique des exploitations agricoles de taille moyenne. Pour examiner les potentielles stratégies d'accumulation et identifier les trajectoires agricoles, nous avons combiné l'analyse des modalités d'accès et d'usage des terres agricoles avec l'identification des pratiques des exploitations agricoles familiales, organisations socio-économiques de base (Benoit-Cattin, 2007). Pour que nos résultats permettent la mise en évidence de profils d'exploitations ayant accumulé des terres, nous avons considéré les exploitations ayant au moins 3 hectares de terres possédées. Ce seuil a été fixé en prenant en compte les statistiques agricoles disponibles, qui estimaient que $69,8 \%$ des exploitations disposent d'un domaine foncier dont la taille est comprise entre 1 à 5 hectares (ANSD, 2014).

L'enquête, réalisée en 2016 et 2017 , a intégré deux phases distinctes :

- une première phase exploratoire a consisté à recenser les exploitations agricoles de villages sélectionnés, pour identifier les superficies possédées ou exploitées, les spéculations cultivées et les moyens matériels de production. L'échantillonnage a été réalisé sur la base du recensement national des villages (accessible en ligne sur basegeo.gouv.sn) et par stratification proportionnelle aux différentes zones agro-écologiques;

- une phase d'enquêtes a été menée auprès d'exploitations sélectionnées selon un seuil minimum de 3 hectares. Ce second échantillon a été obtenu par stratification proportionnelle à la densité de population des zones agroécologiques (Tab. 1).

La première phase d'enquête a été réalisée auprès de 140 villages et 8914 exploitations agricoles. La deuxième sélection n'a retenu que $15,7 \%$ des exploitations, soit 1399 (Fig. 2). Compte tenu de l'objectif de l'analyse, qui est de mettre en évidence un rapport entre superficies possédées et profils d'exploitations, nous avons défini des classes de superficies possédées grâce à une analyse statistique combinant l'analyse des quartiles et des déciles (Tab. 2).

\section{Résultats}

\subsection{Pratiques foncières et profils des chefs d'exploitations étudiées}

Selon les résultats de l'échantillonnage, $62 \%$ des exploitations de plus de 3 hectares se concentrent dans les zones du Bassin arachidier et de la Casamance. Les classes [3-5[ et [10-17[ hectares correspondent chacune à $21 \%$ des observations, la classe [5-10[ à $48 \%$ et la dernière classe compte $10 \%$ des observations (Tab. 2). Concernant le profil sociodémographique des exploitants, l'enquête montre que $81 \%$ d'entre eux sont nés au village et y résident (catégorie 1 : nés au village), $16 \%$ n'y sont pas nés mais y ont passé toute leur vie (catégorie 2 : résidents naturalisés ou intégrés dans le tissu social), et $3 \%$ ne sont pas nés dans le village et n'y 
Tableau 1. Comparaison synthétique des zones agro-écologiques considérées.

Table 1. Summary comparison of the agro-ecological areas considered.

\begin{tabular}{|c|c|c|c|}
\hline Zone agro-écologique & Caractéristiques administratives & Climat & Systèmes de production \\
\hline Niayes & $\begin{array}{l}\text { Bande de terre de } 180 \mathrm{~km} \text { de long et } \\
\text { de } 5 \text { à } 30 \mathrm{~km} \text { de large sur quatre } \\
\text { régions administratives de Dakar à } \\
\text { Saint-Louis }\end{array}$ & $\begin{array}{l}\text { Précipitations abondantes de } 500 \text { à } \\
300 \mathrm{~mm} / \mathrm{an}\end{array}$ & $\begin{array}{l}\text { Production agricole périurbaine } \\
\text { intensive : } 80 \% \text { de la production } \\
\text { nationale en horticulture }\end{array}$ \\
\hline Bassin arachidier & $\begin{array}{l}\text { Zone historique au centre-ouest du } \\
\text { pays }\end{array}$ & $\begin{array}{l}\text { Précipitations comprises entre } 500 \text { et } \\
1000 \mathrm{~mm} / \mathrm{an}\end{array}$ & $\begin{array}{l}\text { Cultures pluviales de mil et arachide } \\
\text { (essentiel de la production nationale) }\end{array}$ \\
\hline Casamance & Zone la plus méridionale & $\begin{array}{l}\text { Climat soudano-guinéen, } \\
\text { précipitations supérieures à } 800 \mathrm{~mm} / \\
\text { an }\end{array}$ & $\begin{array}{l}\text { Activités rizicoles (bas-fonds) } \\
\text { associées aux cultures pluviales et } \\
\text { petits périmètres maraîchers et } \\
\text { horticoles }\end{array}$ \\
\hline
\end{tabular}

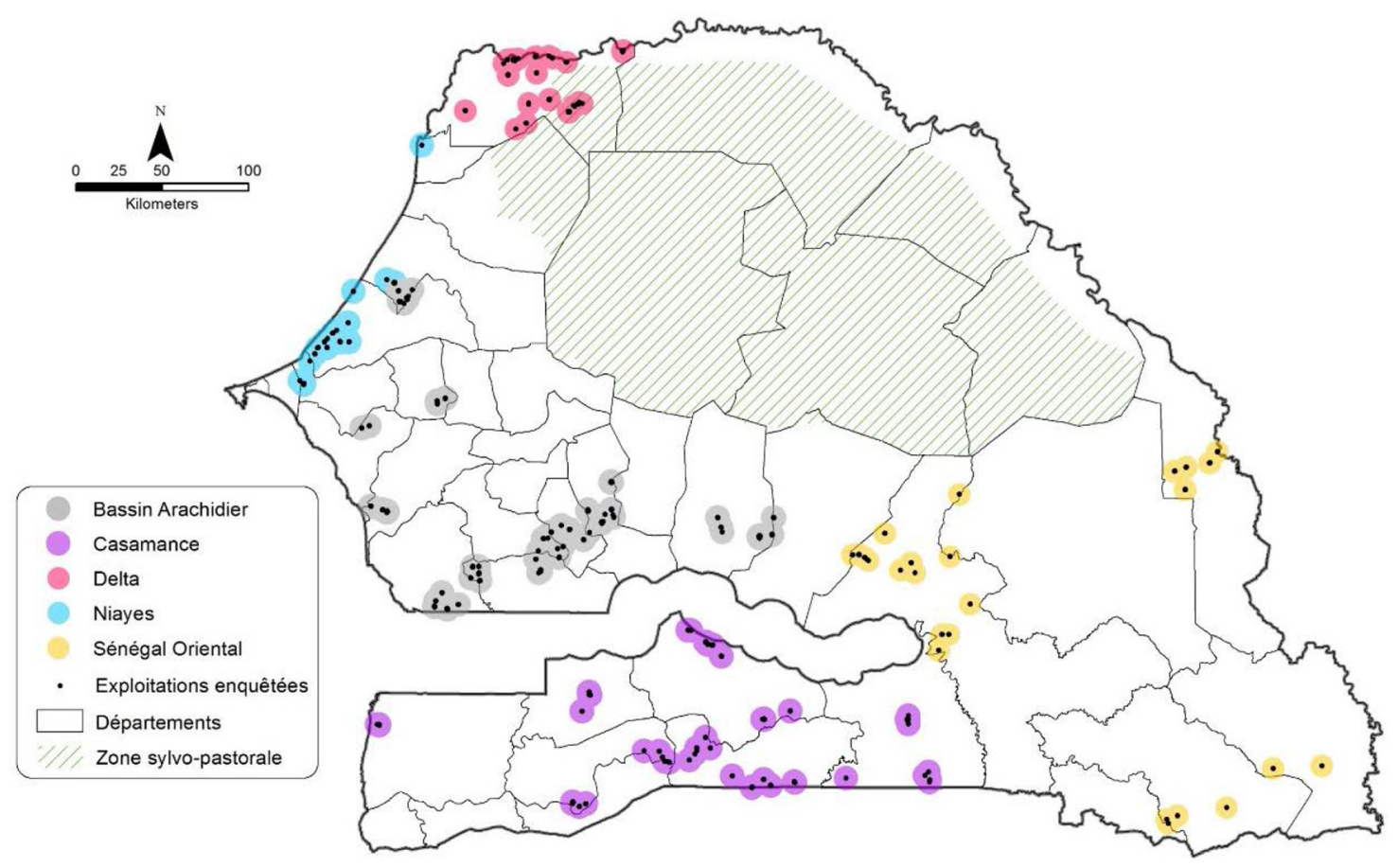

Fig. 2. Localisation des exploitations étudiées.

Fig. 2. Location of the farm surveyed.

résident pas (catégorie 3: exploitants non résidents). Les exploitants de la catégorie 1 possèdent et cultivent $80 \%$ des terres, alors que ceux de la catégorie 2 possèdent et cultivent respectivement 18 et $17 \%$ des terres. Enfin, les exploitants de la catégorie 3 ne possèdent que $2 \%$ des terres et n'en cultivent que $3 \%$. Ces derniers sont principalement recensés dans la zone du Bassin arachidier (Tab. 4). Cela est notamment dû au dynamisme de certains opérateurs privés nationaux qui investissent dans le secteur agricole, et de chefs religieux qui créent de nouveaux villages et écoles coraniques ( $\mathrm{Ba}$ et al., 2018). Nous constatons aussi que la zone du Delta du fleuve Sénégal, qui concentre de nombreux investissements agroindustriels de firmes étrangères, ne connaît que peu d'arrivées d'investisseurs sénégalais non résidents (Tab. 4).

Nous constatons que, quelles que soient leurs superficies, les terres semblent appartenir à des agriculteurs issus des 
Tableau 2. Identification des classes de superficies possédées.

Table 2. Identifying classes of owned land.

\begin{tabular}{lrrrr}
\hline Quartiles et déciles (\%) & 10 & 25 & 50 & 75 \\
Superficie possédée (ha) & 3,5 & 5 & 7 & 10 \\
\hline
\end{tabular}

Tableau 3. Principaux indicateurs définis par classes de superficies.

Table 3. Main indicators defined by area classes.

\begin{tabular}{|c|c|c|c|c|}
\hline & \multicolumn{4}{|c|}{ Classes de superficies possédées (hectares) } \\
\hline Catégorie $1:$ nés au village & 80 & 82 & 81 & 81 \\
\hline Catégorie $2:$ résidents naturalisés & 17 & 15 & 18 & 18 \\
\hline Catégorie 3 : exploitants non résidents & 3 & 3 & 1 & 1 \\
\hline Non familiale à plein temps & $5[0,31]$ & $3[0,04]$ & $5[0,02]$ & $15[0,01]$ \\
\hline Non familiale temporaire & $43[2,73]$ & $58[0,69]$ & $55[0,25]$ & $22[0,01]$ \\
\hline \multicolumn{5}{|c|}{ Principale source de mécanisation (pourcentage) } \\
\hline Location/emprunt de tracteurs & 17 & 18 & 20,3 & 22,5 \\
\hline Propres tracteurs & 0,5 & 0,2 & 0,8 & 3,1 \\
\hline Titre foncier & 0,5 & 0,7 & 0,9 & 0,6 \\
\hline Location & 0,6 & 0,5 & 0,6 & 0,2 \\
\hline Bail & 2,4 & 1,6 & 1,3 & 0 \\
\hline Affectation par la commune & 5,1 & 5,4 & 7,5 & 11,4 \\
\hline Sans document juridique & 91,5 & 91,8 & 89,7 & 87,8 \\
\hline
\end{tabular}

localités (Tab. 3). Au-delà de leur origine, les enquêtes permettent aussi de renseigner les types d'emplois des chefs d'exploitations. Ainsi, il apparait que $89 \%$ des terres appartiennent à des chefs d'exploitations en auto-emploi agricole.

Les exploitations étudiées sont caractérisées par le recours à une main-d'œuvre très majoritairement familiale, ainsi que par un faible niveau de mécanisation. En effet, les exploitations utilisent principalement une main-d'œuvre familiale, appuyée par des employés temporaires. À la différence de ce qui est démontré dans d'autres études (Sitko et Jayne, 2014 ; Anseeuw et al., 2016; Jayne et al., 2016), l'augmentation des superficies cultivées ne s'accompagne pas d'un recours accru à une maind'œuvre non familiale à temps plein (Tab. 3, main-d'œuvre par hectare). La mécanisation se traduit principalement par l'usage de matériels attelés : en moyenne plus de $72 \%$ des exploitants interrogés utilisent leurs propres animaux (chevaux, ânes, bœufs), avec un écart-type très faible de 1,4 sur les différentes classes de superficies considérées. Les effets de classes se constatent sur la location ou l'emprunt d'animaux pour les travaux au champ, ainsi que pour l'accès à la traction motorisée. En effet, plus les classes de superficies augmentent, plus les exploitations remplacent la location d'animaux par l'acquisition de tracteurs (Tab. 3). Le recours à l'emploi hors cercle familial ne se fait que dans les zones du Delta et du Bassin arachidier, où l'intensité des productions et l'importance des surfaces cultivées nécessitent périodiquement l'utilisation d'une main-d'œuvre externe à la famille.

\subsection{Statut foncier et modalités d'accès à la terre}

Sur l'échantillon considéré, plus de $90 \%$ des terres sont sous occupation coutumière (sans document juridique) et seulement $6 \%$ font l'objet d'une affectation foncière par la commune. Cela confirme qu'en milieu rural, la majeure partie des acteurs n'ont pas de droits formalisés (avec un acte administratif ou un titre de propriété) sur les terres occupées. La reconnaissance sociale constitue leur seule garantie ou sécurité sur ces terres. Les baux et titres fonciers, principaux droits fonciers réels, ne concernent respectivement que $1,4 \%$ et $0,6 \%$ des terres occupées. Des différences existent dans les différentes zones agro-écologiques (Tab. 4). Par exemple, la zone du Delta enregistre une importante proportion d'affectations foncières et comparativement moins de terres sans documents juridiques. Dans cette zone, de nombreux projets de développement accompagnent les collectivités locales et les exploitants agricoles pour formaliser leurs occupations foncières traditionnelles. Le même constat s'observe en désagrégeant les résultats par classes de superficies possédées (Tab. 3). Pour toutes les classes, l'occupation coutumière est largement représentée (entre 87 et $92 \%$ des superficies 
Tableau 4. Principaux indicateurs définis par zones agro-écologiques.

Table 4. Main indicators defined by agro-ecological zones.

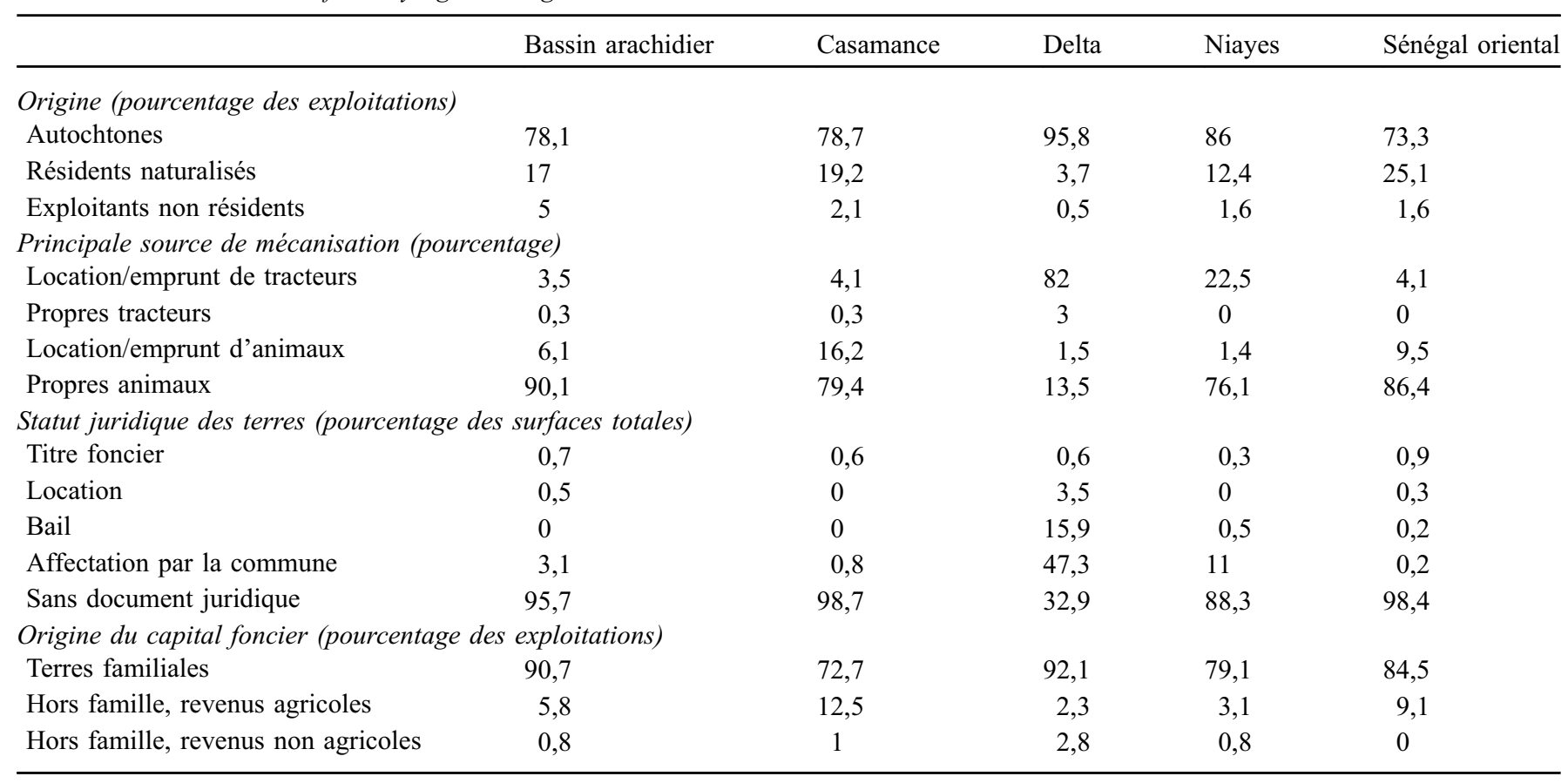

référencées), alors que très peu d'acteurs disposent de droits réels sur les terres. L'héritage et le don de terre restent les modalités privilégiées pour l'accès à de futures terres $(46 \%$ des exploitants interrogés), alors que la location ou l'achat sont envisagés par respectivement $25 \%$ et $20 \%$ des exploitants interrogés.

Les affectations foncières effectuées par les collectivités locales concernent principalement des superficies relativement limitées. Dans les cas où le statut juridique de la terre a changé, $68,75 \%$ des chefs d'exploitations interrogés déclarent avoir bénéficié d'une affectation foncière obtenue au niveau de la commune, alors que $28 \%$ d'entre eux ont eu recours à une régularisation. Cette pratique de sécurisation réalisée par les collectivités locales, quoi qu'en dehors des cadres juridiques, a pour objectif de formaliser les occupations informelles à la demande des exploitants. Les titres fonciers et les baux sont quant à eux plus marginaux (moins de $2 \%$ ).

L'examen des facteurs qui permettent aux acteurs de posséder des terres montre que l'acquisition de terres se fait quasi exclusivement au sein du patrimoine familial, à l'exception de $7,3 \%$ des chefs d'exploitations interrogés pour lesquels l'acquisition s'est faite fait en dehors du cercle familial par achat (principalement dans la zone des Niayes), par location (dans la zone du Bassin arachidier) ou par don (dans la zone du Delta). Seuls $1 \%$ des exploitants interrogés ont investi des revenus issus d'activités non agricoles pour accéder à la terre. Les origines du capital foncier ne sont pas les mêmes en fonction des zones agro-écologiques (Tab. 4). On observe que les investissements réalisés à partir d'activités non agricoles sont très faibles et ne concernent principalement que la zone du Delta et la zone de la Casamance. Cette dernière est marquée par la présence de migrants (autorités religieuses et opérateurs économiques) provenant principalement du Bassin arachidier.

\section{Discussion}

Des études récentes réalisées en Afrique mettent en évidence une accumulation foncière au profit d'élites urbaines et rurales, souvent non issues du monde agricole (Sitko et Jayne, 2014; Anseeuw et al., 2016; Jayne et al., 2016). Les résultats de nos enquêtes au Sénégal montrent qu'en termes de profils, les chefs des exploitations agricoles de plus de 3 hectares possédés sont majoritairement issus de la localité d'origine, cultivent des terres familiales sous occupation coutumière et investissent dans l'agriculture des revenus issus de cette même activité. Globalement, les exploitations étudiées ne semblent pas inféodées à une dynamique de gentrification, caractérisée par l'émergence d'une catégorie d'investisseurs non issus du monde agricole (López-Morales, 2018). Nos résultats mettent aussi en évidence certaines tendances concernant les pratiques foncières. Depuis les années 2000, les politiques publiques d'inspiration libérale ont appuyé une dynamique d'intensification agricole à travers la promotion du secteur agro-industriel. Dans la littérature, ces politiques sont souvent accompagnées d'une volonté de formalisation de droits individuels considérés comme un prérequis du développement de la compétitivité des exploitations agricoles. À l'échelle nationale, ce phénomène semble rester marginal, la majorité des terres restant sous occupation coutumière. Ces particularités pourraient s'expliquer par le fait qu'après l'indépendance du pays, les autorités ont décidé de privilégier une appropriation communautaire de la terre. Depuis 1964, le Sénégal a ainsi fait le choix d'une "sécurité foncière» sans propriété privée par la décapitalisation de la terre. Exposé par Loehr (2012), le principe de décapitalisation associe un droit d'usage précaire à la terre, révocable pour cause d'intérêt général ou public, en vue d'éviter la marchandisation du foncier et les stratégies rentières. En théorie, la décapitalisation 
faciliterait l'accès à la terre pour les plus vulnérables, notamment les communautés pratiquant des usages coutumiers, au-delà de toute considération d'efficience et de recherche de profit. Cette caractéristique du foncier sénégalais pourrait expliquer les faibles niveaux d'appropriation privative et de gentrification observés dans l'étude.

Comme le référencent certains auteurs, ces particularités peuvent aussi s'expliquer par le rôle déterminant des organisations professionnelles agricoles (de Janvry et Sadoulet, 2004 ; Mercoiret, 2006). Dès les années 2000, le Sénégal a notamment été fortement impliqué dans la création du Réseau des organisations paysannes et des producteurs agricoles d'Afrique de l'Ouest. Ce réseau contribue aux débats sous-régionaux sur les enjeux de sécurisation foncière des exploitations familiales en ouvrant des cadres de dialogue. Au niveau national, le Conseil national de concertation et de coopération des ruraux (CNCR), confédération de fédérations paysannes créée en 1993, est moteur depuis 2015 de l'Observatoire national de la gouvernance foncière, outil de diagnostic et de plaidoyer pour la sécurisation foncière des exploitations familiales. Le CNCR accompagne aussi ces exploitations à s'engager sur la voie de l'entreprenariat au travers des actions de son bras technique et fournisseur de services, l'Association sénégalaise pour la promotion du développement par la base. Celle-ci favorise ainsi l'entreprenariat agricole par l'appui à la production agricole et la sécurisation foncière, ainsi que le développement de mécanismes de solidarité. Les organisations de producteurs, et notamment le CNCR, continuent de jouer un rôle majeur dans la conception des politiques publiques, comme la loi d'orientation agro-sylvo-pastorale adoptée en 2004 qui permet la mise en place de fonds d'appui au monde agricole. Cet engagement des structures de la société civile contribue à ce que les exploitations agricoles familiales puissent répondre aux défis du développement agricole.

En 2016, la CNRF a finalisé une proposition de politique foncière soumise au Président de la République, avant d'être dissoute en 2017 (décret $n^{0} 2017-998$ du 16 mai 2017). Ce processus de réforme foncière entamé en 2012 reste donc dans l'attente d'un versant législatif, opérationnalisant la vision, les axes stratégiques et les orientations de réforme proposées. Les choix politiques à venir auront un impact fort sur le devenir du modèle agricole sénégalais, et ils devront prendre en compte certaines particularités territoriales. En effet, si une transition agraire capitaliste n'est pas la norme à l'échelle nationale, nous montrons qu'elle apparaît associée à des dynamiques territoriales singulières dans les zones proches du corridor urbain des régions de Dakar, Thiès et Diourbel, du bassin de production horticole de la zone des Niayes, et des espaces irrigués du Delta du fleuve Sénégal. Identifiés comme hub de production pour les filières prioritaires (riz, horticulture, maraîchage), ces territoires reçoivent, depuis plusieurs décennies, des appuis financiers et techniques qui augmentent une attractivité déjà liée à la proximité des centres économiques, des infrastructures et des services. Ces zones connaissent aussi une progression croissante de la présence de firmes agro-industrielles à capitaux étrangers (Bourgoin et al., 2019), qui suscite de nombreuses critiques et contestations contre l'accaparement des terres, mais génère aussi de l'intérêt quant aux synergies et complémentarités pouvant être développées avec l'agriculture familiale. Dans la mesure où une réforme foncière viendra renforcer une stratégie de transformation de l'agriculture, les organisations professionnelles agricoles engagées dans le processus de réforme devront s'assurer que les orientations choisies en termes de formalisation des pratiques puissent être accompagnées de cadres porteurs d'intérêt général, de cohésion sociale et d'exploitation durable et équitable des ressources foncières.

\section{Conclusion}

Au Sénégal, les espaces agricoles restent majoritairement investis par des agriculteurs issus de leur localité d'origine et du monde agricole en général. Les spécificités du régime foncier sénégalais font que les injonctions de formalisation des droits fonciers portées par le monde du développement n'ont quasiment pas altéré le rapport à la terre des agriculteurs. Cependant, le système foncier actuel connaît de nombreuses limites liées à une faible mise en application des lois et décrets relatifs aux projets de réformes qui se sont succédés depuis 1964, ainsi qu'à la non-prise en compte de dynamiques nouvelles comme l'arrivée de firmes agro-industrielles dans certaines zones attractives du pays. La dissolution de la CNRF n'a pas clôt les débats sur l'orientation de la réforme. Les trajectoires sont multiples entre volonté de décapitaliser les terres, promotion d'une formalisation foncière au profit du marché à travers une immatriculation généralisée des terres et volonté de sécuriser le patrimoine foncier national. Toutefois, au-delà des finalités productivistes, les propositions de réforme devront aussi porter l'ambition d'intégrer les enjeux d'intérêt général, de cohésion sociale, et d'exploitation durable et équitable des ressources foncières.

\section{Références}

ANSD. 2014. Grand recensement 2013, rapport définitif. Agence nationale de la statistique et de la démographie. Dakar, République du Sénégal: Ministère de l'Économie, des Finances et du Plan, 417 p.

Anseeuw W, Jayne T, Kachulen R, Kotsopoulos J. 2016. The quiet rise of medium-scale farms in Malawi. Land 5(19): $22 \mathrm{p}$.

Ba CO, Bourgoin J, Diop D. 2018. Les migrations rurales dans la dynamique migratoire sénégalaise. La fluidité des mobilités internes en réponse aux contraintes locales. Rome: FAO, CIRAD, $28 \mathrm{p}$.

Barral S, Piraux M, Sourisseau J-M., Valette E. 2014. Contribuer aux dynamiques territoriales. In: Sourisseau J-M, ed. Agricultures familiales et monde à venir. Versailles, France: Quae, pp. 111-128.

Benoit-Cattin M. 2007. L'agriculture familiale et son développement durable. Économie rurale 300: 120-123.

Bourgoin J, Valette E, Guillouet S, Diop D, Dia D. 2019. Improving transparency and reliability of tenure information for improved land governance in Senegal. Land 8(3): 17 p.

CNRF. 2016. Document de politique foncière. Dakar, Sénégal: Commission nationale de réforme foncière, $35 \mathrm{p}$.

de Janvry A, Sadoulet E. 2004. Organisations paysannes et développement rural au Sénégal. Washington DC, USA: Banque mondiale, $94 \mathrm{p}$.

FONGS. 2013. Les exploitations familiales sénégalaises investissent et se modernisent. Dakar, Sénégal: Fédération des organisations non gouvernementales du Sénégal, $69 \mathrm{p}$.

Jayne TS, Chamberlin J, Headey DD. 2014. Land pressures, the evolution of farming systems, and development strategies in Africa: a synthesis. Food Policy 48: 1-17. 
Jayne TS, Chamberlin J, Traub L, Sitko N, Muyanga M, Yeboah FK, et al. 2016. Africa's changing farm size distribution patterns: the rise of medium-scale farms. Agricultural Economics 47: 197-214.

Loehr D. 2012. Capitalization by formalization? - Challenging the current paradigm of land reforms. Land Use Policy 29: 837-845.

López-Morales E. 2018. A rural gentrification theory debate for the Global South? Dialogues in Human Geography 8(1): 47-50.

Mercoiret M-R. 2006. Les organisations paysannes et les politiques agricoles. Afrique contemporaine 217(1): 135-157.

NEPAD. 2003. Comprehensive African agriculture development program. Pretoria, South Africa: New Partnership for African Development, $116 \mathrm{p}$.
RCSA. 2017. Revue conjointe du secteur agricole 2017. Dakar, Sénégal: Direction de l'analyse, de la prévision et des statistiques agricoles (DAPSA), $128 \mathrm{p}$.

Sitko N, Jayne TS. 2014. Structural transformation or elite land capture? The growth of "emergent" farmers in Zambia. Food Policy 48: 194-202.

Sourisseau J-M, Bosc P, Fréguin-Gresh S, Bélières J, Bonnal P, Le Coq $\mathrm{J}$, et al. 2012. Les modèles familiaux de production agricole en question. Comprendre leur diversité et leur fonctionnement. Autrepart 62(3): 159-181.

Sourisseau J-M. 2014. Agricultures familiales et mondes à venir. Versailles, France: Quae, 360 p.

Citation de l'article : Bourgoin J, Diop D, Dia D, Sall M, Zagré R, Grislain Q, Anseeuw W. 2020. Regard sur le modèle agricole sénégalais : pratiques foncières et particularités territoriales des moyennes et grandes exploitations agricoles. Cah. Agric. 29 : 18. 